\title{
Treatment of recurrent metastatic uterine leiomyosarcoma of the spine: a multimodality approach using resection, radiosurgery, and chemotherapy
}

\author{
Michael J. Strong, MS, MPH, ${ }^{1-3}$ Trevor Rosenlof, BS, ${ }^{1}$ Siddhartha Padmanabha, MD, ${ }^{4}$ \\ Roy S. Weiner, MD, ${ }^{1,3,5}$ Lee Roy Morgan, MD, PhD, ${ }^{6}$ and Marcus L. Ware, MD, PhD ${ }^{7}$ \\ ${ }^{1}$ Tulane University School of Medicine; ${ }^{2}$ Department of Pathology, Tulane University; ${ }^{3}$ Tulane Cancer Center, New Orleans; \\ ${ }^{4}$ Department of Radiation Oncology, East Jefferson General Hospital, Metairie; ${ }^{5}$ Department of Medicine, Section of Hematology \\ and Medical Oncology, Tulane University School of Medicine; ${ }^{6}$ DEKK-TEC, Inc.; and ${ }^{7}$ Department of Neurosurgery, Ochsner \\ Medical Center, New Orleans, Louisiana
}

\begin{abstract}
The authors describe the case of a patient who initially presented with uterine leiomyosarcoma (LMS) that later metastasized to the spine. The patient was treated at another institution for her primary uterine LMS, undergoing resection followed by adjuvant chemotherapy. After several years of disease remission, the patient presented in January 2011 to the authors' institution with recurrent uterine LMS metastatic to the spine, which has been treated with multiple therapeutic modalities in a combination of surgery, radiosurgery, and chemotherapy. As a result of this approach, the patient has been progression free for 35 months since her presentation (April 2011 to March 2014). We herein describe our experience treating this patient with recurrent uterine LMS of the spine and suggest that patients with recurrent uterine LMSs should be considered for treatment using a multimodality approach with emphasis on enrollment into clinical trials.

http://thejns.org/doi/abs/10.3171/2015.1.SPINE14640
\end{abstract}

KEY WORDS uterine leiomyosarcoma; spinal metastasis; recurrence; radiosurgery; chemotherapy; 4-demethyl-4-cholesteryloxycarbonylpenclomedine; DM-CHOC-PEN; multimodality treatment; oncology

$\mathrm{U}$ TERINE leiomyosarcoma (LMS) is a rare, aggressive smooth muscle malignancy of the uterus, comprising only $1 \%$ of all uterine malignancies. ${ }^{12}$ Due to the infrequency of uterine LMSs and CNS/spinal involvement, as well as to the lack of agreement on the most optimal therapeutic approach to treating, 5-year survival rates vary from $0 \%$ to $75 \%$. The 5 -year survival rate for Stage I uterine LMS is approximately $50 \%-70 \%$, but the rate becomes dismal, $0 \%-22 \%$, in the setting of advanced disease. . $12,13,16,18,23,26$ Although local control after hysterectomy is good, many patients die of disease at a distant site. The reported risk of recurrence is relatively high, with rates ranging from $45 \%$ to $73 \% .{ }^{6}$ Distant metastatic lesions generally occur in the lungs, liver, kidney, brain, and skin, and there is limited involvement with bony structures, such as the spine. ${ }^{4}$ In fact, there is very little information in the current literature about uterine LMS and spinal involvement, and there is no clear consensus about how to best proceed.

We report on a case of recurrence of a uterine LMS to the lumbosacral spine. In a review of the literature, we found only 5 other published reports of a spinal lesion as the first recurrence of metastatic uterine LMS, and none of the cases involved the sacral region..$^{4,21,22,25}$ Spinal involvement can be highly morbid and fatal, requiring individualized multimodality treatments. The goal of this report is to present our experience with combined multimodality therapies to treat this rare recurrent malignancy. 


\section{Case Report}

\section{Clinical History}

This 52-year-old woman was diagnosed in 2007 with a uterine LMS and underwent total abdominal hysterectomy and bilateral salphingo-oophorectomy (TAHBSO) for complete tumor resection at an outside institution. Four years later she presented to our institution. Figure 1 depicts the clinical timeline for this patient. On gross examination, the primary tumor was relatively large (10 $\mathrm{cm}$ ) with irregular borders. Histopathological examination revealed extensive coagulative necrosis with 20 mitotic figures per $10 \mathrm{hpf}$, suggesting a high proliferation index. Surface marker staining showed the presence of estrogen and progesterone receptors on roughly $40 \%$ of the tumor cells. The patient was subsequently enrolled into a Phase II trial of adjuvant letrozole at the outside institution and remained clinically stable during the course of the study until the disease progressed approximately 3.5 years after her initial surgery (December 2010).

\section{Metastatic Spread to the Spine}

In December 2010, the patient presented with progressive transient shooting pains in her left posterior thigh and leg. She also developed a deep throbbing pain in her left buttock, making it difficult to sit. Magnetic resonance imaging revealed an enhancing tumor in the lumbosacral epidural space, but additional imaging of the brain, cervical spine, and thoracic spine demonstrated no other disease at that time. The patient underwent spinal debulking surgery with an L-5 laminectomy at the same outside institution.

\section{First Recurrence of Spinal Uterine LMS}

Shortly after the patient underwent spinal tumor debulking, she presented to our institution and requested to be enrolled into our open Phase I drug trial (DTI-021) with 4-demethyl-4-cholesteryloxycarbonyl-penclomedine (DM-CHOC-PEN), a novel anticancer drug being studied as a treatment for metastatic cancer to the nervous system. ${ }^{15,29}$ As a prerequisite for enrolling into our study, MRI was performed, which showed evidence of tumor recurrence in the spine (Fig. 2A and B). The patient was offered additional surgery and radiation therapy but declined both at that time. Since she declined surgery and radiation ther- apy, and since there is no established standard of care for patients with uterine LMS, she was enrolled in the Phase I study to receive DM-CHOC-PEN (course schedule: 39 $\mathrm{mg} / \mathrm{m}^{2}$ to be repeated every 21 days) in early January 2011.

\section{Second Recurrence of Spinal Uterine LMS}

Unfortunately, after the end of her first course of treatment, the patient presented with new-onset left lower-extremity pain and paresthesias, which progressed to weakness and numbness. She also experienced several days of urinary hesitancy. Lumbar MRI showed evidence of tumor growth, with an extramedullary enhancing mass that measured $5.2 \times 5.1 \mathrm{~cm}$, with effacement and right upward displacement of the thecal sac (Fig. 2C and D). As a result of the imaging evidence of further tumor progression, she was taken out of the Phase I study.

\section{Salvage Treatment With Staged Complex Spine Surgery Followed by Radiosurgery and Chemotherapy}

Our multidisciplinary team, consisting of a neurosurgeon, radiation oncologist, and medical oncologist, evaluated the patient after she was taken out of the Phase I study. After consultation with the patient and family, we devised a plan for maximal safe resection followed by chemotherapy and radiosurgery of the resection bed. The plan also included pharmacokinetic studies to assess the chemotherapeutic sensitivity of the tumor and intratumoral concentrations of DM-CHOC-PEN. The patient was taken to the operating room for tumor debulking and L5-S1 and S-2 laminectomies via a standard approach during continuous nerve root monitoring. The tumor appeared fibrous in nature, adherent to the dura mater and exiting nerve roots, and was of moderate vascularity. Based on these characteristics, we were limited to a subtotal resection. Tumor that was easily separated from the exiting nerve roots was safely removed. Samples of fresh viable tumor tissue were sent to our laboratory for pharmacokinetic studies, including assessment of the tumor's chemotherapeutic sensitivity and levels of DM-CHOC-PEN within the samples. Residual tumor was left in areas where the lesion adhered to the exiting nerve roots and in the S1-2 foramina. Postoperative MRI confirmed residual tumor with enhancement seen abutting the thecal sac and within the left L5-S1 and S1-2 neural foramina, as well as abnor-

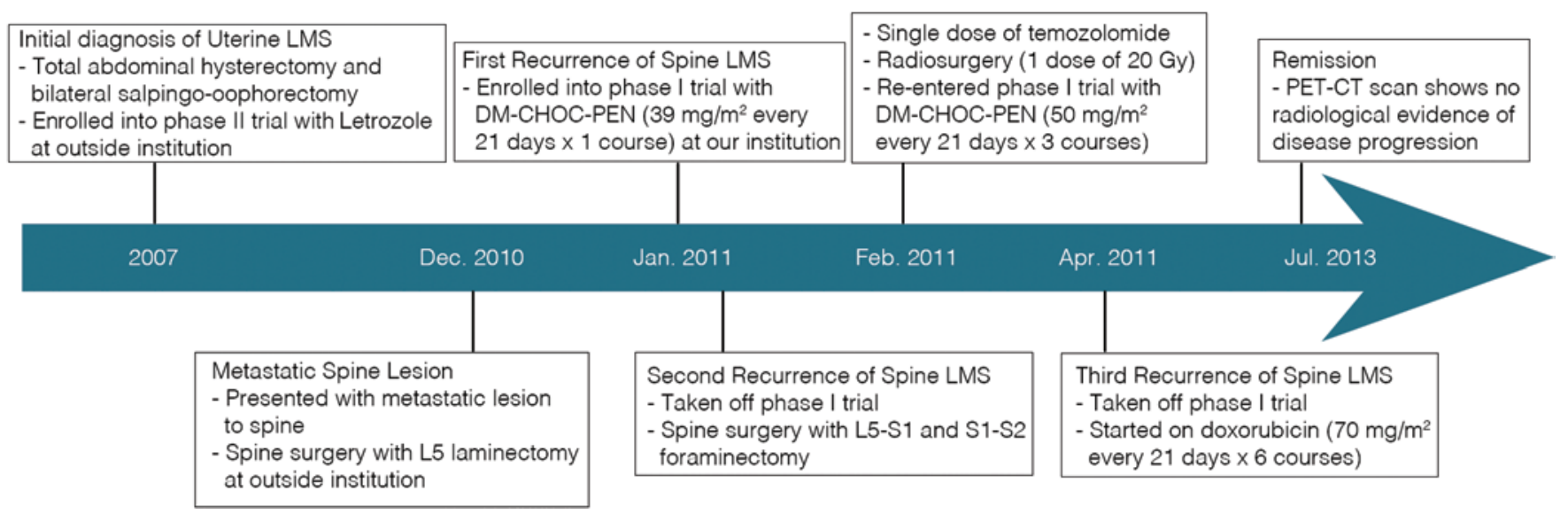

FIG. 1. Clinical timeline of findings and treatments. Figure is available in color online only. 

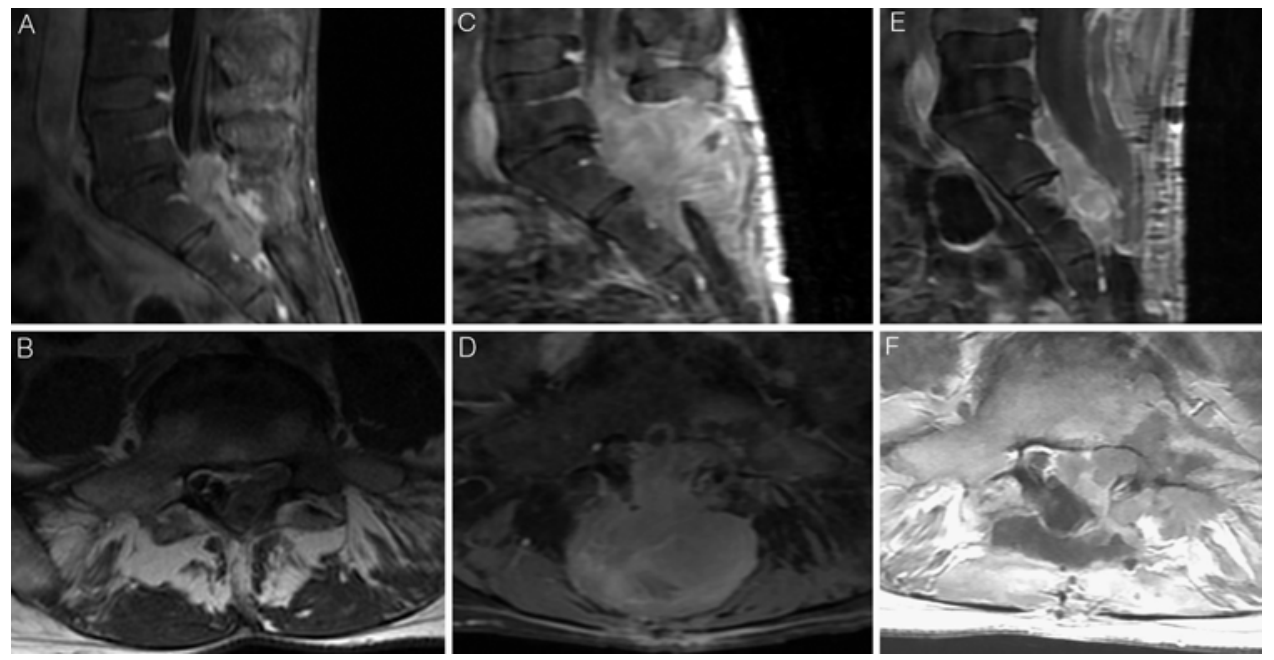

FIG. 2. MR images demonstrating the clinical course of spinal uterine LMS tumor progression. Sagittal (A) and axial (B) T1-weighted images of the patient prior to entrance into DM-CHOC-PEN study. Sagittal (C) and axial (D) T1-weighted images after the patient's first course of DM-CHOC-PEN resulting in the second tumor recurrence. Sagittal (E) and axial (F) T1-weighted images after the patient received multimodality treatment including 3 courses of DM-CHOC-PEN resulting in the third recurrence.

mal enhancement of the medial sacrum. Immediately after surgery her symptoms were worse, but these symptoms improved to baseline several weeks later.

Three weeks after partial surgical debulking, the patient was treated with a single course of adjuvant temozolomide (TMZ) prior to undergoing radiosurgery therapy. Although the patient's tumor was not chemosensitive to TMZ (Table 1), TMZ was chosen because of its synergistic relationship with radiation. , $27,28,30$ Since sarcomas, including uterine LMSs, are typically radioresistant, ${ }^{5,9}$ our rationale was to make the metastasis more radiosensitive, as demonstrated with other tumors such as malignant gliomas. ${ }^{2,24}$ The tumor bed was then treated concomitantly with a single 20-Gy dose of conformational radiation (Fig. $3)$. The patient tolerated this procedure well, with transient urinary hesitancy that was gradually improving and weakness in dorsiflexion of the left foot that unfortunately was not improving to baseline.

\section{Assessment of Tumor Chemotherapy Sensitivity and Levels of} DM-CHOC-PEN

As part of the patient's care, pharmacokinetic studies

\section{TABLE 1. Drug sensitivities for uterine LMS}

\begin{tabular}{lc}
\hline \multicolumn{1}{c}{ Drug } & $\mathrm{IC}_{50}(\mu \mathrm{g} / \mathrm{ml})$ \\
\hline DM-CHOC-PEN & $0.5 \pm 0.01$ \\
\hline Actinomycin D & $0.5 \pm 0.02$ \\
\hline BCNU & $0.9 \pm 0.1$ \\
\hline Cis-platinum & $1.5 \pm 0.1$ \\
\hline doxorubicin & $0.7 \pm 0.1$ \\
\hline TMZ & $>3.0$ \\
\hline HOOI & $0.8 \pm 0.2$ \\
\hline A-007 & $0.3 \pm 0.2$ \\
\hline
\end{tabular}

A-007 = 4,4'-dihydroxybenzophenone-2,4-dinitrophenylhydrazone (a control marker for drug sensitivity; $<1 \mu \mathrm{g} / \mathrm{mL}$ is considered a chemo-sensitive tumor); $\mathrm{BCNU}=$ 1,3-bis(2-chloroethyl)-1-nitrosourea; $\mathrm{HOOI}$ = 4-hydroperoxyifosamide. were performed to assist with the patient's treatment plan. Tumor tissue obtained at the time of the second debulking was portioned to determine chemotherapeutic sensitivity and intratumoral concentrations of DM-CHOC-PEN. Briefly for the chemotherapeutic assay, primary explant cultures were established to perform an in vitro tumor colony-forming assay to measure the lesion's sensitivity to standard and experimental chemotherapeutic agents (Table 1). Each value in Table 1 represents the average of 5 doses $(0.1-10 \mu \mathrm{g} / \mathrm{ml})$ evaluated in triplicate. In addition, A-007 (4,4'-dihydroxybenzophenone-2,4-dinitrophenylhydrazone), which is an experimental cytotoxic agent, was used to identify drug sensitivity. A ratio of cytotoxicity for A-007 versus each drug was compared with the sensitivity of the individual drugs (Table 1). Tumors with an $\mathrm{IC}_{50}$ for A-007 in concentrations $<1 \mu \mathrm{g} / \mathrm{ml}$ are consider chemotherapy sensitive. ${ }^{14}$ The tumor tissue from this patient showed sensitivity to a number of agents including DM-CHOC-PEN (Table 1). Finally, the measurement of intratumoral DM-CHOC-PEN drug concentration was determined by high-performance liquid chromatography.

We found that explanted tumor tissue was sensitive to DM-CHOC-PEN in our in vitro chemosensitivity assay, despite the fact that the patient received DM-CHOC-PEN at a relatively low dose $\left(39 \mathrm{mg} / \mathrm{m}^{2}\right)$ in vivo. Furthermore, the bioavailability of DM-CHOC-PEN demonstrated that
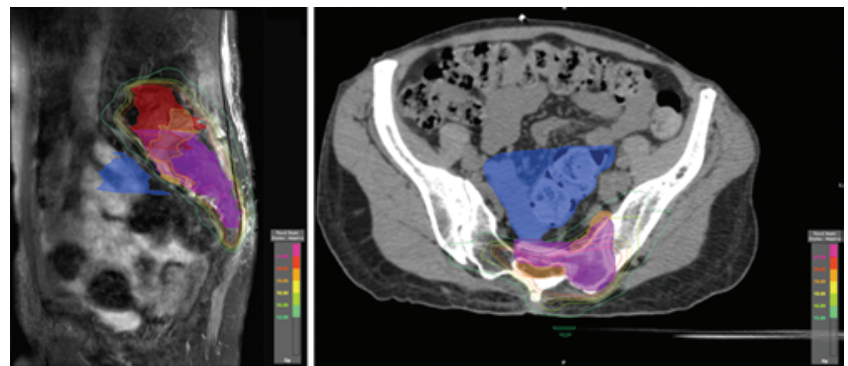

FIG. 3. Radiosurgical dose distribution map shown in sagittal (left) and axial (right) views. Figure is available in color online only. 
there was adequate uptake of the drug with concentrations of 119-192 ng/1 $\mathrm{g}$ of tumor tissue from multiple sites 21 days after the last administration of DM-CHOC-PEN. Taken together, the results of our pharmacokinetic studies suggested the patient might respond at a higher dose of DM-CHOC-PEN.

\section{Third Recurrence of Spinal Uterine LMS}

Upon completion of surgery, chemotherapy, and radiosurgery, the patient again requested to reenter the Phase I DM-CHOC-PEN study. Based on our chemosensitivity and DM-CHOC-PEN bioavailability assays of the patient's tumor, we felt confident about reenrolling her into our Phase I clinical trial. She reentered the study and received a higher initial drug dose $\left(50 \mathrm{mg} / \mathrm{m}^{2}\right.$ compared with $39 \mathrm{mg} / \mathrm{m}^{2}$ ) per treatment protocol. After her third course of treatment, MRI showed evidence of tumor progression, with increased enhancement in the sacrum but without the mass effect seen previously (Fig. 2E and F). The patient was subsequently removed from the drug trial.

\section{Remission}

After exiting the drug trial in April 2011, the patient was started on doxorubicin $\left(70 \mathrm{mg} / \mathrm{m}^{2}\right.$ every 21 days for 6 courses) and then reevaluated for disease progression. She continues to be followed by her oncologist and is clinically stable without evidence of progression. Follow-up imaging showed reduction in tumor mass and enhancement (Fig. 4A and B). The patient's most recent PET CT scan (Fig. 4C) showed no evidence of disease, and her period of progression-free survival has exceeded 30 months.

\section{Discussion}

Uterine LMS is a rare, aggressive malignancy affecting the smooth muscle layer of the uterus, and it has a relatively high recurrence rate even when aggressive therapy is used. When uterine LMS recurs, its treatment has
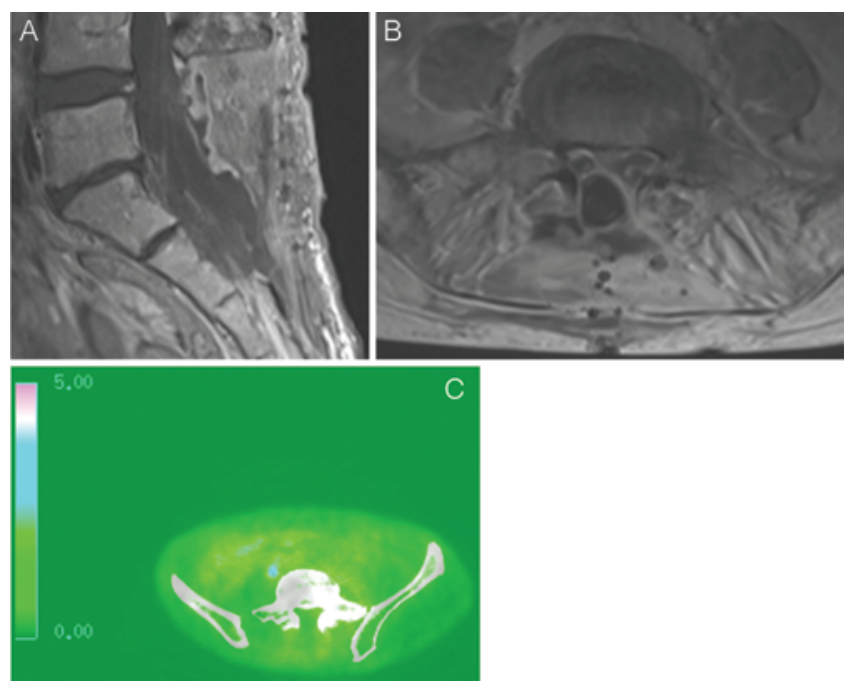

FIG. 4. Posttreatment images. Lumbosacral sagittal (A) and axial (B) T1-weighted MR images reflecting more than 30 months of progressionfree survival. Axial PET CT scan (C) also demonstrating no evidence of disease progression. Figure is available in color online only. often been palliative because of the disease's high mortality rate. Specifically, tumors showing the cluster of $>20$ mitotic figures/10 hpf, positive Ki 67 staining, large tumor size $(>10 \mathrm{~cm})$, and negative BCL2 staining have a markedly unfavorable prognosis. ${ }^{3}$ We present a case of recurrent metastatic uterine LMS to the spine, with evidence of remission since 2011 after a multimodality therapeutic approach.

In our case, a complete resection of the uterine LMS was not possible because of the tumor's location and its intimate involvement with the dura and exiting spinal nerve roots. Although negative margins are warranted to attain optimal outcomes in cases of metastatic spinal sarcomas, including LMSs, achieving a gross-total tumor resection is hindered by the tumor anatomy and the desire to preserve neurological function. ${ }^{1}$ In cases involving a residual metastatic tumor of the spine, postoperative radiation therapy may be helpful in improving local control but not overall survival. ${ }^{11,20}$ Furthermore, in the European Organization for Research and Treatment of Cancer Gynecological Cancer Group Study (EORTC-GCC) 55874 trial, ${ }^{19}$ unlike sarcomas in general, uterine LMS-specific local control rates were not optimal, with similar local failure rates seen between the fractionated radiotherapy arm (20\%) and observational arm (24\%). Despite limited success using conventional fractionated radiotherapy to treat metastatic spinal sarcomas, radiosurgery has been shown to be effective in achieving good local control rates of $84 \%-88 \%$ in some studies. 5 , Therefore, radiosurgery was chosen over fractionated radiotherapy for this patient.

Efficacious chemotherapy for uterine LMS with spinal and CNS involvement is still lacking, as illustrated by the poor prognosis seen in cases of metastatic uterine LMS, even when detected early. To address this growing concern, recent efforts by several groups have increased uterine LMS patients' participation in clinical trials; however, patients with CNS involvement are often excluded.

There have been several novel chemotherapeutic agents tested in recent years in Phase I/II trials, but limited or no activity is frequently reported..$^{17}$ Combination therapy has demonstrated improved response rates, with the two most active treatment regimens being gemcitabine plus docetaxel and doxorubicin with or without ifosfamide. ${ }^{17}$ Studies have demonstrated a $48 \%$ response rate in patients treated with doxorubicin and ifosfamide ${ }^{10}$ and a $53 \%$ overall response rate for patients treated with gemcitabine and docetaxel. ${ }^{7}$ With the high preponderance of hematological spread and the relatively low chemotherapeutic response rates for uterine LMSs, new chemotherapeutic agents are warranted. Although it is still in the early stages of drug trial, DM-CHOC-PEN shows promise as an effective adjuvant chemotherapeutic drug for recurrent uterine LMSs because of its ability to penetrate and concentrate in malignant tissue, as demonstrated by our DM-CHOC-PEN intratumoral bioavailability assessment.

In the present case, the exact reason for the long-term progression-free survival is unclear. Our patient's treatment plan was complex and included surgery followed by radiosurgery and three different modes of chemotherapy (TMZ, DM-CHOC-PEN, and doxorubicin). Any one of these treatment modalities could have contributed to our 
patient's improved outcome, but a combination of these agents is more likely to explain the successful result. It is noteworthy that despite our aggressive regimen with multiple modes of treatment, the patient was able to tolerate her treatments well.

Lastly, we realize that radiosurgery alone has been associated with good local control ${ }^{5,9}$ and that the enhancement seen on follow-up MRI may have been pseudo-progression and/or an adverse radiation effect from the radiosurgery. Our early experience with DM-CHOC-PEN, however, has shown a preponderance of pseudo-progression prior to longer-term progression-free survival (unpublished data). Therefore, on the basis of the presence of DM-CHOCPEN at levels of 119-192 ng/1 $\mathrm{g}$ of tumor tissue after the initial single dose $\left(39 \mathrm{mg} / \mathrm{m}^{2}\right)$, we feel confident that there was probably drug present after the 3 courses of $50 \mathrm{mg} /$ $\mathrm{m}^{2}$, playing a possible contributory role in this patient's progression-free survival. In addition, doxorubicin was started 21 days after the third dose of DM-CHOC-PEN was administered, and the combination of these two drugs is unknown. Both drugs have the novel ability of inducing reduction-oxidation-cycling cytotoxic changes in tumors and may be synergistic. To understand the efficacy of DM-CHOC-PEN on uterine LMSs, additional studies in a clinical trial platform are needed.

\section{Conclusions}

Uterine LMS is a rare and aggressive malignancy that very rarely spreads to the spine. Treatment should aim to preserve function. In the present case, the patient responded well to a multimodal therapeutic approach involving subtotal resection followed by single-fraction radiosurgery and a combination chemotherapy regimen, which included chemotherapeutic sensitivity testing. Patients with recurrent uterine LMSs should be considered for treatment using a multimodality approach with emphasis on enrollment in clinical trials.

\section{References}

1. Bilsky MH, Boland PJ, Panageas KS, Woodruff JM, Brennan MF, Healey JH: Intralesional resection of primary and metastatic sarcoma involving the spine: outcome analysis of 59 patients. Neurosurgery 49:1277-1287, 2001

2. Carlson BL, Grogan PT, Mladek AC, Schroeder MA, Kitange GJ, Decker PA, et al: Radiosensitizing effects of temozolomide observed in vivo only in a subset of O6-methylguanine-DNA methyltransferase methylated glioblastoma multiforme xenografts. Int J Radiat Oncol Biol Phys 75:212-219, 2009

3. D’Angelo E, Espinosa I, Ali R, Gilks CB, Rijn Mv, Lee CH, et al: Uterine leiomyosarcomas: tumor size, mitotic index, and biomarkers Ki67, and Bcl-2 identify two groups with different prognosis. Gynecol Oncol 121:328-333, 2011

4. Elhammady MSA, Manzano GR, Lebwohl N, Levi AD: Leiomyosarcoma metastases to the spine. Case series and review of the literature. J Neurosurg Spine 6:178-183, 2007

5. Folkert MR, Bilsky MH, Tom AK, Oh JH, Alektiar KM, Laufer I, et al: Outcomes and toxicity for hypofractionated and single-fraction image-guided stereotactic radiosurgery for sarcomas metastasizing to the spine. Int J Radiat Oncol Biol Phys 88:1085-1091, 2014

6. Giuntoli RL II, Metzinger DS, DiMarco CS, Cha SS, Sloan
JA, Keeney GL, et al: Retrospective review of 208 patients with leiomyosarcoma of the uterus: prognostic indicators, surgical management, and adjuvant therapy. Gynecol Oncol 89:460-469, 2003

7. Hensley ML, Maki R, Venkatraman E, Geller G, Lovegren M, Aghajanian C, et al: Gemcitabine and docetaxel in patients with unresectable leiomyosarcoma: results of a phase II trial. J Clin Oncol 20:2824-2831, 2002

8. Koukourakis GV, Kouloulias V, Zacharias G, Papadimitriou C, Pantelakos P, Maravelis G, et al: Temozolomide with radiation therapy in high grade brain gliomas: pharmaceuticals considerations and efficacy; a review article. Molecules 14:1561-1577, 2009

9. Laufer I, Iorgulescu JB, Chapman T, Lis E, Shi W, Zhang $\mathrm{Z}$, et al: Local disease control for spinal metastases following "separation surgery" and adjuvant hypofractionated or high-dose single-fraction stereotactic radiosurgery: outcome analysis in 186 patients. J Neurosurg Spine 18:207-214, 2013

10. Leyvraz S, Zweifel M, Jundt G, Lissoni A, Cerny T, Sessa C, et al: Long-term results of a multicenter SAKK trial on highdose ifosfamide and doxorubicin in advanced or metastatic gynecologic sarcomas. Ann Oncol 17:646-651, 2006

11. Mahdavi A, Monk BJ, Ragazzo J, Hunter MI, Lentz SE, Vasilev SA, et al: Pelvic radiation improves local control after hysterectomy for uterine leiomyosarcoma: a 20-year experience. Int J Gynecol Cancer 19:1080-1084, 2009

12. Major FJ, Blessing JA, Silverberg SG, Morrow CP, Creasman WT, Currie JL, et al: Prognostic factors in early-stage uterine sarcoma. A Gynecologic Oncology Group study. Cancer 71 (4 Suppl):1702-1709, 1993

13. Mayerhofer K, Obermair A, Windbichler G, Petru E, Kaider A, Hefler L, et al: Leiomyosarcoma of the uterus: a clinicopathologic multicenter study of 71 cases. Gynecol Oncol 74:196-201, 1999

14. Morgan LR, inventor; Dekk-Tek, Inc., assignee: Methods to predict tumor response to therapy. US patent 5,270,172. December 14, 1993

15. Morgan LR, Struck RF, Waud WR, LeBlanc B, Rodgers AH, Jursic BS: Carbonate and carbamate derivatives of 4-demethylpenclomedine as novel anticancer agents. Cancer Chemother Pharmacol 64:829-835, 2009

16. Naaman Y, Shveiky D, Ben-Shachar I, Shushan A, MejiaGomez J, Benshushan A: Uterine sarcoma: prognostic factors and treatment evaluation. Isr Med Assoc J 13:76-79, 2011

17. O'Cearbhaill R, Hensley ML: Optimal management of uterine leiomyosarcoma. Expert Rev Anticancer Ther 10:153169,2010

18. Pautier P, Genestie C, Rey A, Morice P, Roche B, Lhommé $\mathrm{C}$, et al: Analysis of clinicopathologic prognostic factors for 157 uterine sarcomas and evaluation of a grading score validated for soft tissue sarcoma. Cancer 88:1425-1431, 2000

19. Reed NS, Mangioni C, Malmström H, Scarfone G, Poveda A, Pecorelli S, et al: Phase III randomised study to evaluate the role of adjuvant pelvic radiotherapy in the treatment of uterine sarcomas stages I and II: an European Organisation for Research and Treatment of Cancer Gynaecological Cancer Group Study (protocol 55874). Eur J Cancer 44:808-818, 2008

20. Reichardt P: The treatment of uterine sarcomas. Ann Oncol 23 (Suppl 10):x151-x157, 2012

21. Robbins LL: Roentgenologic demonstration of spinal metastases from leiomyosarcoma of the uterus. Arch Surg 47:463-467, 1943

22. Schjott-Rivers E: Sarcoma of the uterus. Acta Obstet Gynecol Scand 28:418-425, 1949

23. Soumarová R, Horová H, Seneklová Z, Ruzicková J, Horová I, Budíková M, et al: Treatment of uterine sarcoma. A survey of 49 patients. Arch Gynecol Obstet 266:92-95, 2002 
24. Stupp R, Mason WP, van den Bent MJ, Weller M, Fisher B, Taphoorn MJB, et al: Radiotherapy plus concomitant and adjuvant temozolomide for glioblastoma. N Engl J Med 352:987-996, 2005

25. Takemori M, Nishimura R, Sugimura K, Mitta M: Thoracic vertebral bone metastasis from uterine leiomyosarcoma. Gynecol Oncol 51:244-247, 1993

26. Van Dinh T, Woodruff JD: Leiomyosarcoma of the uterus. Am J Obstet Gynecol 144:817-823, 1982

27. van Rijn J, Heimans JJ, van den Berg J, van der Valk P, Slotman BJ: Survival of human glioma cells treated with various combination of temozolomide and X-rays. Int J Radiat Oncol Biol Phys 47:779-784, 2000

28. Wedge SR, Porteous JK, Glaser MG, Marcus K, Newlands ES: In vitro evaluation of temozolomide combined with Xirradiation. Anticancer Drugs 8:92-97, 1997

29. Weiner R, Ware M, Friedlander P, Gordon C, Saenger Y, Mahmood T, et al: A first-in-humans Phase I cancer clinical trial for 4-demethyl-4-cholesteryloxycarbonylpenclomedine (DM-CHOC-PEN) in humans. Cancer Res 73:1185, 2013 (Abstract)
30. Wick W, Wick A, Schulz JB, Dichgans J, Rodemann HP, Weller M: Prevention of irradiation-induced glioma cell invasion by temozolomide involves caspase 3 activity and cleavage of focal adhesion kinase. Cancer Res 62:1915-1919, 2002

\section{Author Contributions}

Conception and design: Ware, Strong, Weiner, Morgan. Acquisition of data: Rosenlof. Analysis and interpretation of data: all authors. Drafting the article: Ware, Strong, Rosenlof, Padmanabha. Critically revising the article: Ware, Strong, Padmanabha, Weiner, Morgan. Reviewed submitted version of manuscript: Ware, Strong, Padmanabha, Weiner, Morgan. Approved the final version of the manuscript on behalf of all authors: Ware. Study supervision: Ware.

\section{Correspondence}

Marcus L. Ware, Department of Neurosurgery, Ochsner Medical Center, 1514 Jefferson Highway, New Orleans, LA 70121. email: marcusware@gmail.com. 